\title{
Conciencia emocional en la formación del profesorado a través de elementos musicales y visuales: uso de la lengua como medio de expresión de las emociones
}

Noemí Martín ${ }^{1}$ y Eva Jiménez ${ }^{1,2 *}$

(1) Facultad de Ciencias Sociales y de la Comunicación, Dpto. de Educación y Humanidades, Universidad Europea de Madrid, C. Tajo s/n, Villaviciosa de Odón, 28670, Madrid, España. (correo-e: noemimartyn@gmail.com)

(2) Vicerrectorado de Profesorado e Investigación, Unidad de Calidad y Compliance, Universidad Europea de Madrid,

C. Tajo s/n, Villaviciosa de Odón, 28670, Madrid, España. (correo-e: eva.jimenez@universidadeuropea.es)

${ }^{*}$ Autor a quien debe ser dirigida la correspondencia

Recibido Dic. 20, 2019; Aceptado Feb. 18, 2020; Versión final Abr.3, 2020, Publicado Ago. 2020

\begin{abstract}
Resumen
El objetivo del trabajo presentado es potenciar el desarrollo de la conciencia emocional dentro del aula, mediante elementos musicales y visuales a través del uso de la lengua como medio de expresión emocional, con la intención de integrar la competencia emocional como elemento transversal dentro del proceso de enseñanza-aprendizaje. Se realizó una actividad de identificación y gestión emocional a través de la expresión lingüística de las emociones provocadas durante la escucha de una melodía y la observación de una imagen. El análisis de los datos determina dificultades en los futuros docentes a la hora de identificar sus emociones, así como un elevado nivel de interés sobre la integración de este contenido dentro del proceso educativo. Se concluye que sería necesario ampliar la formación previa de docentes en competencia emocional con la intención de lograr una formación completa e integral del alumnado, y, por tanto, la mejora de la calidad educativa.
\end{abstract}

Palabras clave: inteligencia emocional; expresión emocional; conciencia emocional; música en el aula

\section{Emotional awareness in teacher formation through musical and visual elements: use of language as means of expressing emotions}

\begin{abstract}
The objective of this study is to enhance the development of emotional awareness within the classroom through musical and visual elements as a means of emotional expression. This is with the intention of integrating emotional competence as a transversal element within the teaching-learning process. An activity of identification and emotional management is performed through the linguistic expression of emotions caused during the listening of a melody and the observation of an image. Data analyses show difficulties for future teachers in identifying their emotions. The results also show the high level of interest on the integration of this content in the educational process. In conclusion, it is necessary to expand previous training of teachers on emotional competence to achieve complete and comprehensive student training and to improve educational quality.
\end{abstract}




\section{INTRODUCCIÓN}

La misión con la que nace la educación es el desarrollo integral de los individuos para formar personas adaptadas a la sociedad en la que vivimos. En este sentido, se debe buscar el desarrollo cognitivo a través de conocimientos, pero sin olvidar que la educación requiere del fomento de competencias sociales y emocionales, ya que va a facilitar la adaptación al medio de vida: la sociedad. Bajo esta perspectiva nace la Inteligencia Emocional (en adelante IE) que llegó al mundo educativo para quedarse, con la intención de aportar el conocimiento que potenciase el desarrollo en los estudiantes a nivel afectivo, social y emocional. Según señala Bisquerra (2005), la IE se entiende como "un proceso educativo, continuo y permanente, que pretende potenciar el desarrollo emocional como complemento indispensable del desarrollo cognitivo, constituyendo ambos los elementos esenciales del desarrollo de la personalidad integral' (p.96).

A nivel pedagógico, se pretende convertir la IE en aprendizaje de emociones como parte del proceso de enseñanza-aprendizaje en cualquier etapa educativa, con el fin de concienciar formar alumnos con un aprendizaje competencial e integral. No obstante, hoy en día queda mucho trabajo por realizar y retos que asumir en base a la educación emocional como parte de la práctica docente dentro del aula. Es por ello que, el presente estudio de investigación tiene como objeto principal comprobar los resultados de una intervención Ilevada a cabo para desarrollar la conciencia emocional mediante elementos musicales y visuales utilizando la lengua como medio de expresión, en concreto utilizando la expresión escrita. El interés de esta intervención nace de las dificultades y problemas que se observan en el aula, donde actualmente no se presta atención suficiente al desarrollo de la IE, y en concreto al fomento de la conciencia emocional, reflejándose en nuestra realidad social, en la que encontramos personas con carencias en la detección y gestión emocional, lo cual tiene un efecto negativo en su desarrollo integral con el resto de la sociedad.

Una de las causas que produce este déficit en la formación integral de los estudiantes es la falta de formación en el profesorado sobre la educación emocional, ya que, actualmente, son muchos los docentes que no han tenido la oportunidad de adquirir y desarrollar mecanismos que les faciliten la identificación y gestión de sus emociones. Esto a su vez, conlleva la imposibilidad de la transmisión del aprendizaje emocional a los alumnos, lo cual obtiene como resultado la existencia de alumnos sin formación completa e integral. Respecto a esto, Bisquerra (2005) señala la ausencia en programas de formación de maestros y considera que los docentes tendrían que tener una formación inicial que proporcionara un "bagaje sólido en materia de emociones y sobretodo en competencias emocionales" (p.100), además de llevar a cabo una formación continua del profesorado, de manera permanente. Además, la falta de desarrollo de esta competencia en el aula demuestra carencias en la adquisición de herramientas de detección y gestión de emociones, lo cual se demuestra en la realización y resolución de actividades que no tienen base emocional en su esencia.

De igual manera, las consecuencias que se transcriben de esto lo hacen en forma de dificultades en los estudiantes a nivel personal, los cuales no van a poder gestionarlos de manera correcta ya que no tienen adquirida esa competencia ni instrumentos para poder afrontarlos. Por todo esto, es necesario e ineludible abordar el proceso de enseñanza-aprendizaje atendiendo a cuestiones emocionales para que los estudiantes se beneficien de este aprendizaje, ya que la emoción y la razón están continuamente interaccionando y por ello no se puede llegar a comprender la situación educativa como un proceso que no tiene en cuenta cómo se siente el alumnado (Mujica, Inostroza y Orellana, 2018).

La relevancia del tema se encuentra en las finalidades de la intervención, que, por un lado, pretende desarrollar conciencia emocional en las aulas y, por otro lado, formar continuadamente al profesorado para mejorar la transmisión de aspectos emocionales a los estudiantes. Esto origina estudiantes competenciales a nivel emocional, a través del trabajo en el aula con tareas y actividades que aborden cuestiones emocionales. De esta manera, se va a favorecer que los estudiantes dispongan de instrumentos suficientes para poder gestionar posibles dificultades o problemas que aparezcan en su vida, a nivel personal. En este aspecto, Mujica, et al. (2018) sostienen que, llevando a cabo la práctica docente, es posible abordar factores personales y socioculturales que intervienen en cuestiones emocionales de los estudiantes "para de esta forma elaborar estrategias que lo ayuden a desarrollar buenas emociones en forma sistemática, proporcionando las experiencias que contribuyan a afrontar los factores que promueven un inadecuado emocionar" (p. 121). Por tanto, en este trabajo se presenta una intervención con resultados cualitativos y cuantitativos, cuya finalidad es desarrollar la conciencia emocional en el aula a través de la realización de una actividad de identificación y gestión emocional, usando elementos musicales y visuales, además de la lengua como vehículo de expresión de las emociones.

Tras los estudios realizados por Mayer y Salovey (1990) sobre IE, es Goleman (1995) quien lleva a cabo un profundo trabajo sobre esta nueva idea, poniendo de relevancia el interés que suscita no sólo en el ámbito de la educación, sino recalcando la importancia que esta tiene en las diferentes relaciones personales. A día de hoy, cada vez es más frecuente encontrar autores que realizan estudios relacionados con la IE y su importancia dentro del contexto educativo. Para entender esta importancia Cohen (2006) señala que debería 
reformularse el objetivo de la educación con la intención de modificar la finalidad del aprendizaje académico, poniendo atención en las competencias emocionales, sociales y también éticas. De esta manera, el autor defiende que, desarrollando estas competencias, se mejorará las habilidades que establecen el origen de una sociedad democrática, así como la calidad de vida. Por todo ello, entendemos la IE como la capacidad del ser humano para afrontar y gestionar adecuadamente las emociones, por tanto, no se intenta definir esta inteligencia como una forma de terminar con las emociones, sino de saber dirigirlas y equilibrarlas. Debido a estas razones, es necesario desarrollar esta capacidad en los estudiantes dentro del aula, como elemento transversal del proceso de enseñanza-aprendizaje.

\section{OTROS ANTECEDENTES}

Hay ciertos aspectos que requieren ser analizados para el contexto de este trabajo: docentes competenciales a nivel emocional, música y emociones, inteligencia emocional y rendimiento académico y la lengua como medio de expresión emocional.

\section{Docentes competenciales a nivel emocional}

Como aspecto fundamental, se requieren docentes con formación previa cualificada con el objetivo de poder transmitir un aprendizaje emocional, dotando así a sus estudiantes de las herramientas necesarias para detectar y gestionar sus emociones. De esta manera, Bisquerra (2005) apela a la necesidad de la formación del profesorado para el desarrollo de la IE, proponiendo en su artículo, la implantación de programas de educación emocional para la formación del profesorado en competencias emocionales, señalando de esta forma la importancia que adquiere la formación constante. Este trabajo cobra especial relevancia para este estudio, ya que, persiguiendo uno de nuestros objetivos en esta investigación, establece como punto de partida la formación del profesorado, que ha de adquirirse de manera constante, para el correcto desarrollo de la competencia emocional en el aula con los estudiantes.

Siguiendo con esta línea, Hawkey (2006) estudia la relación que se da en torno a la necesidad de formación del profesorado en la labor tutorial con respecto a la IE, y se valoran las posibles causas de que existan estas carencias emocionales en el campo educativo, en general, y en los docentes, en particular. Al respecto, se aportan algunas reflexiones que apuntan algunas dificultades, y de esta manera razonan algunos aspectos sobre política y la práctica en Gran Bretaña, concluyendo que se puede extrapolar al territorio internacional. Por otra parte, desde Italia, Fabio y Palazzeschi (2008) realizan un estudio sobre la construcción de la IE y su relación con los docentes en este territorio. Los autores determinan la necesidad de llevar a cabo una profunda reflexión sobre la IE en los docentes y continuar realizando estudios de investigación al respecto. Bajo esta misma perspectiva, y centrando su atención hacia la competencia emocional que han desarrollado los docentes, Corcoran y Tormey (2013) llevan a cabo su trabajo. En el mismo afirman que faltan datos sobre la relación que se establece entre la habilidad emocional de los docentes y su desempeño o rendimiento docente. Ante esta problemática reflexionan sobre el rendimiento docente encontrando dificultades entre la comprensión de las emociones y la enseñanza de esta competencia a los estudiantes.

Para concluir este enfoque, y con el fin de alertar sobre la necesidad extrema de incluir como parte de los objetivos mínimos en Educación Obligatoria las competencias emocionales, Palomera, Fernández-Berrocal y Brackett (2008), realizan una revisión sobre investigaciones relativas a competencias emocionales, sobre el funcionamiento personal, social y académico de los estudiantes, así como del bienestar de los docentes. El objeto de este trabajo es probar la necesidad de incrementar las competencias emocionales en los docentes para así impulsar y favorecer tanto el rendimiento laboral como su bienestar, al igual que el de los estudiantes. Finalizan el mismo aportando una propuesta: formación del profesorado como preferente en el aprendizaje, así como la formación constante. Además, los autores insisten en sus conclusiones sobre la exigencia de adoptar decisiones para "ser coherentes [...] con lo que las leyes educativas nos exigen y con el modelo de sociedad europea que perseguimos" (p.449).

En torno a los aspectos señalados, este trabajo ayuda a identificar, una vez más, la necesidad general con la que nos encontramos en las aulas de diferentes etapas de Educación sobre la falta de trabajo de competencias emocionales. En este sentido, es relevante para nuestro estudio, no solo en la detección de la necesidad en formación de los docentes, sino en el fomento del interés por el trabajo de IE dentro del proceso de enseñanza-aprendizaje.

\section{Música y emociones}

Otro de los aspectos especialmente relevantes en el estudio de investigación que aquí se plantea es la importancia que tiene la música en cuanto a su relación y conexión con las emociones. En este mismo sentido Alaminos (2014) realiza un estudio cuya finalidad es comprobar la relación que se da entre las emociones y la música, realizando un estudio con dos grupos de muestra. Los individuos del primer grupo indican el tipo 
de música que elegirían para escuchar dependiendo de un estado de ánimo específico, y los del segundo grupo son los encargados de valorar la consideración de que esas canciones expresan esos estados de ánimo. Así, el autor concluye que, tanto para la música como para las emociones, se da una segunda capacidad oculta. No obstante, el autor concluye indicando cómo se producen emociones a través de la escucha de canciones. Por tanto, el desarrollo de este trabajo permite señalar la relación que se da entre música y respuesta emocional, lo cual sustenta la idea del diseño de la actividad realizada como objeto de estudio de la presente investigación, en la que se utiliza la música para facilitar la aparición de distintas emociones. Por otro lado, Resnicow, Salovey y Repp (2004) reflexionan en su trabajo sobre como dentro de la interpretación musical se realiza la expresión de diferentes emociones, como un aspecto de comunicación no verbal. Para llevar a cabo este estudio, utilizan la prueba de Inteligencia Mayer-Salovey-Caruso para conseguir valorar el grado de identificación, compresión y manejo de las diferentes emociones en diferentes situaciones. De tal manera, concluyen la correlación significativa que se da entre la IE y el reconocimiento de emociones, donde estudiantes universitarios identifican emociones a través de una audición musical.

Siguiendo esta línea en la búsqueda de posibles vínculos entre la IE y aspectos musicales, Schellenberg (2011) lleva a cabo un estudio en el que valora a participantes familiarizados musicalmente y participantes que no lo están. De esta manera lleva a cabo diferentes pruebas donde los valores más positivos en IE los tuvieron los participantes que habían sido entrenados en el mundo musical. De esta manera, a pesar de no poder concluir de manera fehaciente el vínculo entre IE y música en las pruebas que utiliza, el autor afirma como existen algunas evidencias que hacen pensar que se dan asociaciones entre la música y habilidades intelectuales.

\section{Inteligencia emocional y rendimiento académico}

Otra de las perspectivas estudiadas hasta el momento por algunos autores, como Páez y Castaño (2006) o Buenrostro-Guerrero et al. (2012) es la relación que se da entre la IE y el rendimiento académico en los estudiantes, en algunos casos creando controversia entre estos conceptos. Con el propósito de analizar la importancia que tienen las emociones en el contexto educativo y profundizar en la relación que se da entre la IE y el éxito académico de los estudiantes, Swanepoel y Britz (2017) buscan comprobar la relación que se da entre la IE y el rendimiento académico. En el mismo, utilizan la Prueba Adaptativa Computarizada de Potencial de Aprendizaje (LPCAT) y la prueba de Inteligencia Emocional Cuerpo-Mente, desarrollada por Jerabek en 1996. Tras analizar los resultados, se comprueba como de manera significativa se da una relación positiva entre el valor de la IE y el rendimiento académico, pero los autores señalan que se requiere de más investigaciones para poder concluir de manera generalizada estos valores obtenidos.

Siguiendo esta línea, Páez y Castaño (2015) llevan a cabo un estudio de investigación con la intención de realizar la descripción de la IE y estudiar la correspondencia de esta con el rendimiento académico de los alumnos en la etapa de Educación Superior, en concreto, en la Universidad. Concluyen con la significatividad en la correspondencia que se da entre IE y rendimiento académico, sin diferencias en cuanto al género, pero con diferencias entre los programas de estudios universitarios. No obstante, afirman que no se puede determinar de manera clara el rendimiento académico únicamente con el valor de la IE, ya que, en palabras de los propios autores, en el rendimiento académico "inciden diversos factores y no puede explicarse solo por la IE, aunque este sí es uno de los factores a tener en cuenta." (p.280). Este estudio ayuda a fomentar el interés por la IE como parte del proceso enseñanza-aprendizaje en el aula y poder comprobar los beneficios que aporta en el desarrollo de las personas, los cuales se enmarcan dentro de los objetivos de nuestro trabajo de investigación.

\section{La lengua como medio de expresión emocional}

En cuanto a la relación existente entre la IE y su expresión, a través del uso de la lengua, son varios los autores que han llevado a cabo diferentes estudios. Así, Soriano (2016) en su trabajo pretende investigar sobre la interdisciplinariedad de las emociones, ya que señala a todos los campos de conocimiento, incluyendo la lingüística entre las disciplinas. De esta manera, durante su estudio, lleva a cabo una revisión sobre lenguaje metafórico de las emociones o lenguaje emocional literal, entre otros. De tal forma, el autor, con sus propias palabras, concluye que "el lenguaje nos informa sobre cómo representamos nuestro universo emocional. Tanto las palabras que designan emociones como las metáforas que usamos para hablar de ellas encapsulan un auténtico conocimiento enciclopédico sobre qué son y cómo son las emociones" (p.255). Además, señala que el lenguaje emocional tiene multitud de aplicaciones prácticas y establece relaciones con la psicología y neurociencias en el momento en que sería imposible conocer las representaciones emocionales si no se diese una expresión lingüística de las mismas. Los aportes que conlleva este trabajo son especialmente relevantes para nuestro estudio, en el enfoque que aporta al uso de la lengua como medio de expresión emocional, la cual se establece como uno de los pilares fundamentales de la presente investigación. 
Con la intención de dotar a los estudiantes de herramientas que les permitan expresar a través del lenguaje aspectos emocionales, Cruz (2014) lleva a cabo un estudio en el que desarrolla la IE entorno a distintas habilidades y competencias, relacionándolas con el currículum, en concreto de la etapa de Educación Infantil. El objeto de este estudio es desarrollar la I IE a través del uso de la lengua, reflexionando sobre la importancia de la construcción de la personalidad desde el momento del nacimiento. La autora concluye que es fundamental "poner al alcance del alumnado todos los tipos de lenguaje que les sirvan para expresar su mundo emocional, un buen camino para el descubrimiento de las emociones es a través de la expresión lingüística" (p.108). Este aporte es significativo para el presente estudio, ya que demuestra la importancia del uso de la lengua en la expresión emocional, lo cual se enmarca como objetivo principal de esta investigación.

Por su parte, Sucaromana (2012), trabaja en la búsqueda de posibilidades de fomentar y potenciar la IE en la enseñanza y aprendizajes de la lengua, ya que el contenido emocional toma protagonismo en todos los aspectos de la vida, incluyendo la Lengua. Pese a la importancia que esto tiene para el desarrollo personal, la competencia emocional no tiene la consideración que debería en su relación con el aprendizaje de la Lengua. Tomando en cuenta la significatividad de la competencia emocional en el aprendizaje, se podría conseguir una enseñanza más efectiva de la lengua. Por otro lado, Ozlem (2006) aporta una ardua reflexión sobre la relación que se produce entre IE con respecto al aprendizaje de la lengua. En el mismo, el autor aborda el concepto innato de inteligencia y lenguaje en las personas, y de esta manera establece la IE como un tipo de inteligencia social que permite establecer un control de las emociones de uno mismo y de otros con la intención de orientar nuestras acciones. De esta manera, concluye que las personas con un alto desarrollo de la competencia emocional tienen mayor facilidad de aprendizaje en los idiomas.

\section{METODOLOGÍA}

Conocidos los antecedentes, a continuación, se presenta la metodología utilizada en este estudio, detallando los objetivos de la investigación, el diseño metodológico y muestral, así como los instrumentos para la recogida de datos.

\section{Objetivos}

El objetivo del trabajo presentado es potenciar el desarrollo de la conciencia emocional dentro del aula, para lo cual se lleva a cabo una intervención utilizando elementos musicales y visuales que propicien el uso de la lengua como medio de expresión emocional. Tras esta intervención, los objetivos específicos que se proponen son: detectar la necesidad de formación continuada al profesorado sobre IE; mostrar los beneficios percibidos de la IE en el aula y reconocer la IE como elemento trasversal utilizando la lengua como medio de expresión.

\section{Diseño metodológico y muestral}

El diseño metodológico utilizado en esta investigación es mixto. Mediante la realización de una actividad de identificación y gestión emocional, usando la Lengua como vehículo de expresión de las emociones, se obtienen resultados cuantitativos y cualitativos. Por un lado, se utiliza el método de diseño no experimental con un diseño descriptivo, en el que el papel del investigador es el de observar, describir y fundamentar aspectos de la situación que se estudia. Por otro lado, dentro del enfoque cualitativo, se lleva a cabo una intervención, orientada a la comprensión de los resultados. El diseño muestral de este estudio responde a un muestreo no probabilístico por conveniencia. La muestra objeto de estudio (tabla 1) está compuesta por 39 estudiantes de la Universidad Europea de Madrid, de los cuales 22 estudiantes pertenecen al Máster en Formación del Profesorado y 17 alumnos al Máster Universitario en Educación Bilingüe que, tras sus estudios, se convertirán en futuros docentes de diferentes etapas de Educación (Infantil, Primaria o Secundaria):

Tabla 1: Descripción de la muestra.

\begin{tabular}{|l|l|}
\hline Máster en Formación del Profesorado & Máster Universitario en Educación Bilingüe \\
\hline 22 estudiantes de la especialidad de Lengua Castellana & 17 estudiantes \\
\hline Mujeres: 18 & Mujeres: 15 \\
\hline Hombres: 4 & Hombres: 2 \\
\hline $\begin{array}{l}\text { Total \% mujeres: } 84,6 \% \\
\text { Total \% hombres: } 15,3 \% \\
\text { Edades comprendidas entre los } 22 \text { y } 43 \text { años }\end{array}$ \\
\hline
\end{tabular}




\section{Instrumentos}

Para la recogida de datos se elaboran tres instrumentos. El primero de ellos corresponde con la actividad para desarrollar la conciencia emocional, que supuso la construcción de la misma, para lo cual se realizaron diferentes tareas como lo son la búsqueda y elección de la imagen sobre la que trabajar en la actividad, así como una melodía que evoque emociones y redacción del enunciado de la actividad. En concreto, la actividad consistente en la expresión lingüística de las emociones suscitadas por la visualización de una imagen, al tiempo que se escucha una melodía con diferentes frecuencias emocionales, de tal forma que se integra la competencia emocional, como elemento transversal, dentro del proceso de enseñanza-aprendizaje. Los estudiantes deben visualizar la imagen de la Figura 1 con la música de la Banda Sonora Original de "Leyendas de pasión" (James Horner) y expresar lo que sienten mediante el lenguaje escrito, es decir, la palabra.

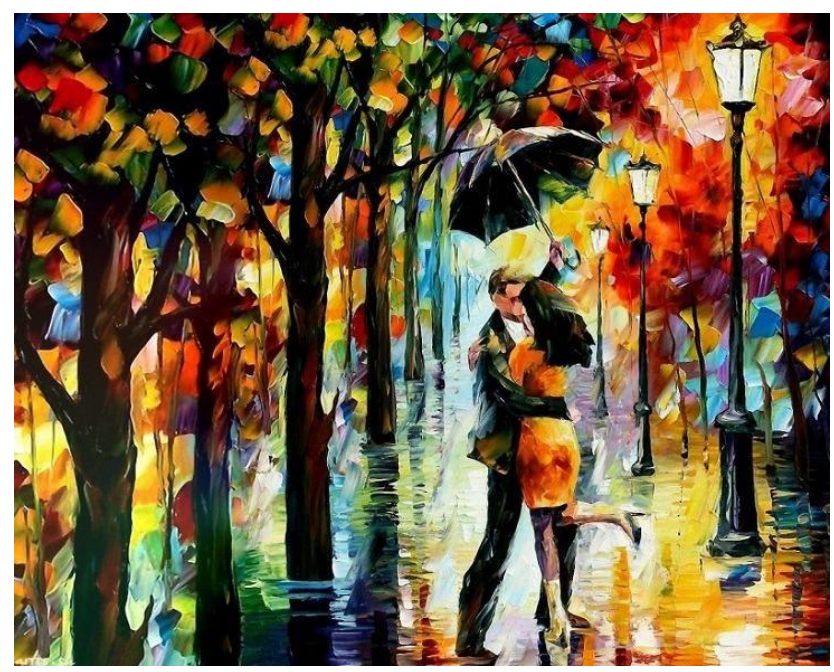

Fig. 1: Bailando bajo la lluvia. Artista: Leonid Afremov

Otro instrumento, de elaboración propia, fue una lista de cotejo que permitiera evaluar la actividad llevada a cabo con los estudiantes sobre el desarrollo de la conciencia emocional, a través del uso de la lengua como medio de expresión. Para la creación de este instrumento se han tenido en cuenta parámetros como el nivel de implicación del participante de la muestra, atendiendo al uso de la lengua y su empleo de la misma en la gestión de sus emociones, recursos lingüísticos empleados o expresiones que hagan referencias a sentimientos o emociones, distribuidos en 6 ítems dicotómicos sobre la detección de la necesidad de formación en IE (tabla 2) y 7 ítems dicotómicos sobre el reconocimiento de la IE utilizando la lengua como elemento transversal (tabla 3).

Por último, se elaboró un cuestionario mixto, ad hoc, de 14 preguntas y transcrito al inglés. Dicho cuestionario no ha sido validado, pero ha permitido estudiar el objetivo previsto y valorar el uso de la lengua como medio de expresión emocional. El cuestionario se compone de preguntas cerradas, como la identificación de emociones (previas, durante, posteriores) a la realización de la actividad; y preguntas de respuesta abierta, relacionadas con la reflexión sobre la experiencia emocional vivida durante la realización de la actividad o los beneficios que pueden aportar la realización de tareas como la que se ha llevado a cabo en esta ocasión.

\section{RESULTADOS Y DISCUSIÓN}

En las siguientes líneas se mostrarán los principales resultados agrupados en tres bloques, atendiendo a los objetivos de estudio.

\section{Necesidad de formación continuada al profesor sobre IE}

Atendiendo a los resultados que nos ofrece la lista de cotejo utilizada en la actividad, con la intención de detectar la necesidad de formación al profesorado, en la tabla 2 se puede comprobar como el $100 \%$ de la muestra se involucró de manera activa y adecuada en la realización de la actividad, a través del uso de la lengua como medio de expresión emocional, lo cual se indicaba en el enunciado de la misma. También puede observarse como más del $55 \%$ de la muestra consigue verbalizar a través de la palabra la emoción que están sintiendo en ese momento y transmitir al lector cómo se siente utilizando un tipo de lenguaje que consigue contagiar la emoción al lector, utilizando recursos lingüísticos, palabras que evocan emociones, cuestiones temporales que incitan al recuerdo, etc. El 54\% es capaz de expresar momentos vividos y/o experiencias personales en su escrito, ayudándose del recurso personal, recuerdos y vivencias propias para conseguir expresar sus emociones de una manera más sencilla, objetivo de la actividad realizada en el estudio. 
Un aspecto a destacar es que el $82 \%$ de los participantes llevan a cabo un texto en el que aportan reflexiones propias, involucrándose activamente en este escrito y evitando, de esta manera, realizar únicamente la descripción de la imagen. Los resultados obtenidos en este trabajo coinciden con Giraldo (2011) en donde afirma que la creación de textos en los que se escriba desde las emociones, pueden "“' [...] mejorar el concepto de sí mismo y logra dinamizarse al definir planes y proponerse estimulantes acciones que contribuyen a obtener una nueva imagen de su ser y de su existir." (p.207), concluyendo, por tanto, la existencia de esta gran fuente de conocimiento de uno mismo en este tipo de textos.

Tabla 2: Resultados evaluados en la lista de cotejo sobre la actividad en IE

\begin{tabular}{|c|c|c|c|}
\hline Objetivos & En el escrito se observa que el estudiante... & SI & NO \\
\hline \multirow{6}{*}{$\begin{array}{l}\text { Detectar la } \\
\text { necesidad de } \\
\text { formación del } \\
\text { profesorado }\end{array}$} & ....se ha involucrado en la realización de la actividad. & $100 \%$ & $0 \%$ \\
\hline & ...utiliza la lengua como medio de expresión emocional & $100 \%$ & $0 \%$ \\
\hline & ...se realiza, únicamente, un texto descriptivo sobre la imagen & $18 \%$ & $82 \%$ \\
\hline & $\begin{array}{l}\text {...verbaliza la emoción que está sintiendo a través del uso de la lengua en el } \\
\text { escrito }\end{array}$ & $56 \%$ & $44 \%$ \\
\hline & ...es capaz de transmitir a lector la emoción que está sintiendo & $59 \%$ & $41 \%$ \\
\hline & $\begin{array}{l}\text {...expresa momentos vividos o experiencias personales, a través del uso de } \\
\text { la lengua como medio de expresión emocional en el texto }\end{array}$ & $54 \%$ & $46 \%$ \\
\hline
\end{tabular}

En lo que respecta al cuestionario, se pregunta a la muestra por las emociones detectadas o identificadas en el momento previo a la realización de la actividad sobre IE en el aula (figura 2) y también se les pregunta por las emociones sentidas tras la actividad (figura 3). Los resultados alcanzados, presentados en la Figura 2, manifiestan emociones positivas y negativas. Entre las emociones positivas podemos destacar como la mayoría de los estudiantes, 33\%, se mostraban cómodos, seguidos de un $10 \%$ que indicaban sentirse motivados, así como un $3 \%$ señalaba curiosidad e intriga. En lo que concierne a las emociones negativas se destaca con un $13 \%$ que los estudiantes se muestran nerviosos, con un $10 \%$ se sienten desganados e indecisos y con un $8 \%$ avergonzados.

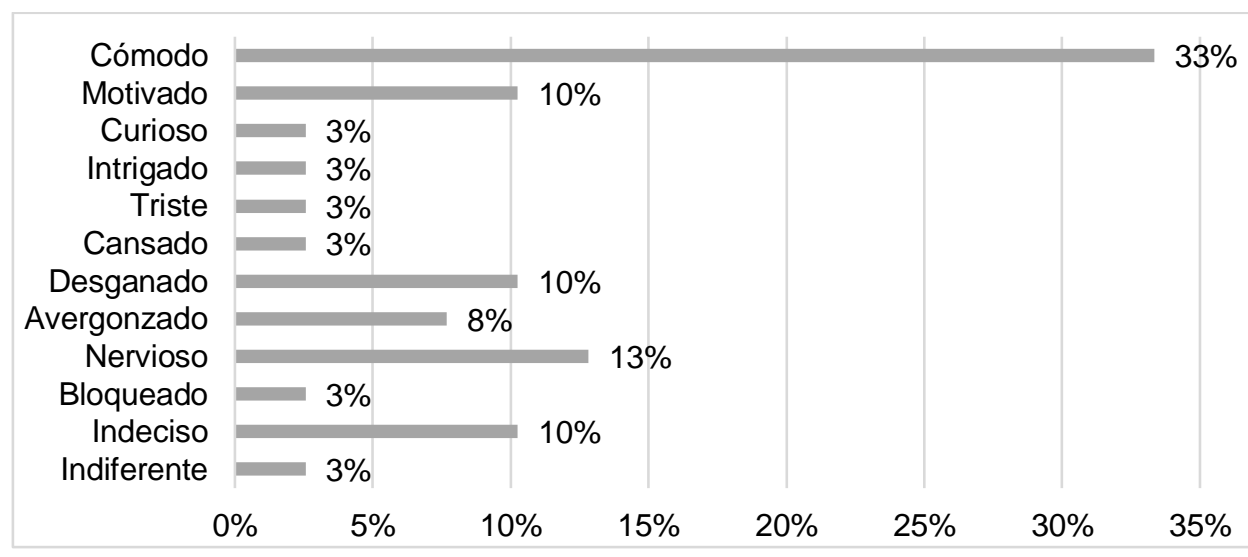

Fig. 2: Emociones antes de la actividad

Tras la actividad, ver Figura 3, es llamativo el cambio significativo en lo que respecta a los sentimientos negativos, pues tan solo un $3 \%$ de los estudiantes dicen que han sentido asco. Las emociones positivas son elevadas, mostrando felicidad y sorpresa tras la actividad (21\% y $10 \%$ respectivamente). Por otro lado, la opción de otros aumenta un $36 \%$, lo que muestra la dificultad existente en la identificación de emoción sentida durante el desarrollo de la actividad; al igual que sucede con la emoción de tristeza, que aumenta un $26 \%$.

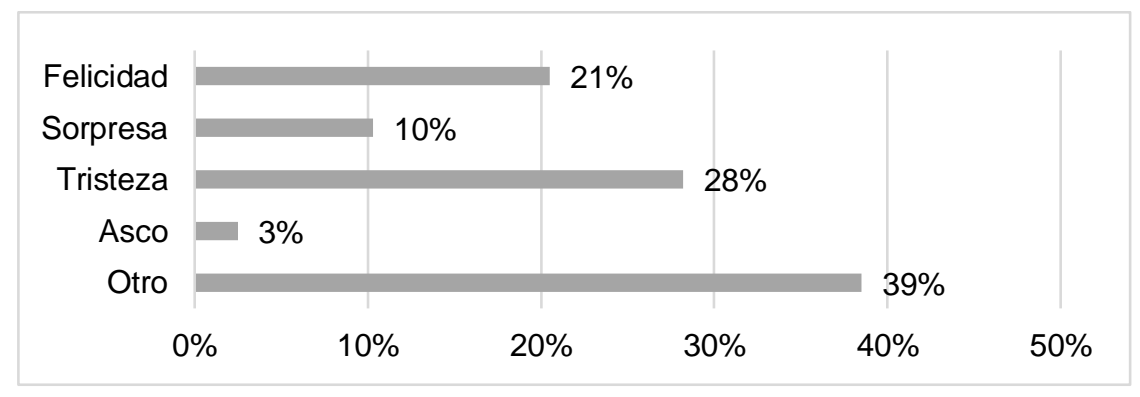

Fig. 3: Emociones después de la actividad 
En cualquier caso, sea cual sea la emoción sentida, el 64\% los participantes muestran que, tras la actividad, han sufrido un cambio en el estado emocional. A pesar de que son unos resultados positivos, en un porcentaje bastante elevado se demuestra la existencia de carencias en la formación básica de los futuros docentes en materia de gestión y expresión emocional, en el momento en que son incapaces de identificar la emoción que han sentido. Según Torrijos, Martín y Rodríguez (2018), coinciden en la importancia que tiene la competencia emocional dentro del proceso en el ámbito educativo, destacando que los docentes deben haber sido preparados para poder ser capaces de transmitir herramientas en materia emocional a sus estudiantes con el fin de que puedan utilizarlas en una mejora de la adaptación o resolución de conflictos, entre otras. De igual manera, concluyen que es necesario seguir potenciando algunos aspectos de educación emocional como es la regulación emocional en los docentes. Análogamente, Escolar, De la Torre, Huelmo y Palmero (2017) en su trabajo afirmar la escasez de programas socio-emocionales destinados para la formación de docentes en el campo emocional, lo cual se ve reflejado y corroborado por los resultados obtenidos en este trabajo.

Con la intención de fomentar el interés del trabajo de IE integrado dentro del proceso de enseñanzaaprendizaje y diseñar procedimientos, procesos y/o actividades para detectar y gestionar las emociones, se pide a la muestra una valoración sobre si la actividad realizada durante este estudio de investigación ha resultado interesante, útil o beneficiosa. Los resultados que se obtienen resultan positivos en un $90 \%$ de la muestra.

\section{Beneficios percibidos de la IE}

Otro de los objetivos que se pretende en esta investigación es mostrar los beneficios que aporta la IE en el aula. Para ello, se propone a la muestra que aporten su opinión sobre los posibles beneficios que aporta el trabajo de la IE dentro del aula. Los resultados, resumidos en la Figura 4, constatan que el $26 \%$ de la muestra, coincide en que uno de los beneficios es la posibilidad de llevar a cabo la expresión emocional, seguido de un $18 \%$ que se refiere al autoconocimiento emocional, señalando así la relevancia que tiene este aspecto para los participantes. Con menor peso, 5\%, cabe destacar, como un beneficio, la posibilidad de que la educación emocional suponga un proceso reflexivo para el estudiante.

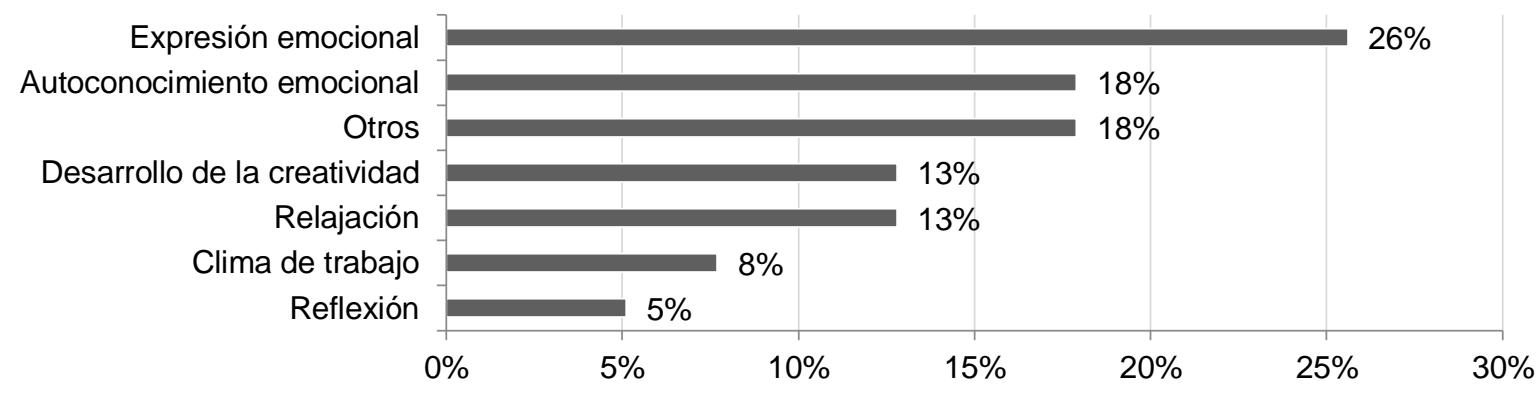

Fig. 4: Beneficios de la IE percibidos por los participantes de la intervención

En este sentido, no son demasiados los trabajos que evalúan de manera práctica los beneficios que tiene el trabajo de la competencia emocional en el aula. No obstante, en consonancia a los resultados obtenidos en este trabajo, Pérez-Escoda, Filella, Alegre y Bisquerra (2012), centrando su atención en el desarrollo de la conciencia emocional en maestros y alumnos, observan una mejora en las competencias emocionales de los participantes, destacando resultados positivos y, por lo tanto, la eficacia del programa de formación que estaban estudiando. De igual manera ocurre con el trabajo de Ozáez (2015) en el que la autora concluye que algunos de los beneficios del trabajo emocional en el aula se reflejan en la adecuada utilización, comprensión y manejo de las emociones para mejorar las relaciones con los demás o superación de dificultades imprevistas que van apareciendo en la vida.

En este mismo sentido, Jennings y Greenberg (2009) en su labor de tomar conciencia sobre los beneficios que aporta el desarrollo de la IE, proponen trabajar en el aula de una manera en el que se desarrolle la competencia social y emocional, dando como resultado un bienestar de los docentes que a su vez va a provocar que se establezcan relaciones adecuadas entre docentes, estudiantes y un excelente clima de trabajo en el aula. Esta implementación del programa de aprendizaje emocional y social propiciarán un fomento del aprendizaje consiguiendo resultados significativos entre los estudiantes. Por último, en el trabajo de Petrides et al. (2006), los autores estudian como beneficia la IE en las relaciones entre los niños durante la etapa escolar. En el estudio participaron estudiantes y docentes, concluyendo que los alumnos con un desarrollo emocional mayor, afirman tener un comportamiento en el que fomentan el bienestar social dentro del aula, sin embargo, los estudiantes con un nivel menor en IE, tienen rasgos de comportamiento que no beneficia al aspecto social. En ambos estudios se puede comprobar como un aspecto señalado de beneficios que aporta el desarrollo de la IE es el nombrado por los autores como bienestar social, tanto en docentes como en estudiantes. 


\section{La IE como elemento trasversal}

A través de la lista de cotejo, se recoge información sobre la relación entre el ámbito lingüístico y la educación emocional en el aula. En la tabla 3 se observa que el $92 \%$ de la muestra utiliza recursos lingüísticos durante la expresión escrita, haciendo uso así de adjetivos, símiles, metáforas o retórica entre otras. En torno al $80 \%$ de los participantes utilizan sustantivos que ayudan a reflejar cuestiones emocionales (como son el recuerdo, Iluvia, sentidos, destino, sonrisa, dolor, nostalgia, etc.), expresiones temporales (pasado, tiempo, algún día, siempre o alguna vez...) y el uso de verbos que evocan emociones en sus escritos (sentir, echar de menos, abrazar, sonreír o querer).

Respecto a la utilización de vocabulario que evoca expresiones sobre emociones o sentimientos, entendiendo estos como amor, felicidad, tristeza, emociones, sentimientos, melancolía, etc., el $77 \%$ refleja alguna expresión de este tipo en su escrito. Respecto a estos resultados, Beck et al. (2012) en el estudio sobre la competencia emocional de los niños, afirman la importancia que está teniendo en los últimos años, teniendo en cuenta su relación también con el lenguaje, a pesar de que esta relación entre la competencia emocional y lingüística no se ha estudiado lo suficiente. A pesar de esto, los autores, concluyen afirmando la correlación entre la Lengua y las emociones, en concreto sobre el vocabulario receptivo y la alfabetización en cuanto al aprendizaje de emociones y conciencia de emociones mixtas.

Por último, en cuanto a la utilización del número y persona, un $64 \%$ utiliza la primera persona del singular o del plural para expresar sus emociones en el escrito, mientras que el $36 \%$ no utiliza la primera persona. Del porcentaje que no utiliza la primera persona, un 33\% utiliza, en su mayoría, la redacción en segunda persona del plural.

Tabla 3: Resultados evaluados en la lista de cotejo sobre la actividad en IE

\begin{tabular}{|c|c|c|c|}
\hline Objetivos & En el escrito se observa que el estudiante... & SI & NO \\
\hline \multirow{7}{*}{$\begin{array}{l}\text { Reconocer la } \\
\text { Inteligencia } \\
\text { Emocional utilizando } \\
\text { la lengua como } \\
\text { elemento } \\
\text { transversal }\end{array}$} & $\begin{array}{l}\text {...utiliza recursos lingüísticos (adjetivos, símiles, metáforas, retórica) en el } \\
\text { escrito. }\end{array}$ & $92 \%$ & $8 \%$ \\
\hline & $\begin{array}{l}\text {...se expresa a través del uso de la primera persona del singular y/o plural } \\
\text { (yo/nosotros). }\end{array}$ & $64 \%$ & $36 \%$ \\
\hline & $\begin{array}{l}\text {...utiliza cualquier otra persona que no sea la primera persona del singular } \\
\text { y/o plural. }\end{array}$ & $33 \%$ & $67 \%$ \\
\hline & $\begin{array}{l}\text {...hace uso de expresiones que hagan referencias a sentimientos o } \\
\text { emociones. (Amor, felicidad, tristeza, sentimiento, melancolía, emociones, } \\
\text { etc.) }\end{array}$ & $77 \%$ & $23 \%$ \\
\hline & $\begin{array}{l}\text {..utiliza palabras como: recuerdo/acuerdo, lluvia, sentidos, rencuentro, } \\
\text { destino, corazón, sonrisa, tiempo, engaño, dolor, nostalgia, conmigo, etc. }\end{array}$ & $85 \%$ & $15 \%$ \\
\hline & $\begin{array}{l}\text {...usa verbos como: sentir, echar de menos, abrazar, sonreír, recordar, } \\
\text { querer, etc. }\end{array}$ & $80 \%$ & $21 \%$ \\
\hline & $\begin{array}{l}\text {...hace uso de expresiones temporales (pasado, momentos, hoy, tiempo, } \\
\text { algún día, siempre, última vez, últimamente, alguna vez, mañana, etc.) }\end{array}$ & $82 \%$ & $18 \%$ \\
\hline
\end{tabular}

Con relación al vínculo que se puede crear entre IE y el ámbito lingüístico, encontramos el trabajo de MarínMoya, Ruiz, Sánchez-Abril y Pagán (2015), que a pesar de tener un enfoque distinto que el que se muestra en este trabajo de investigación, aportan conclusiones de vital importancia para el planteamiento principal entorno al desarrollo de la IE a través del uso de la lengua que aquí se proyecta. En este sentido, los autores afirman haber conseguido unos resultados motivadores y positivos en la dimensión afectivo-emocional, integrando contenidos del currículo y de educación emocional a través del uso de la lengua, lo cual coincide con los resultados obtenidos en esta investigación, pero en esta ocasión enfocada desde la lectura y no desde la escritura. Coincidiendo con este trabajo, Trimmer y Cuddy (2008) Ilevan a cabo un estudio con estudiantes universitarios en el que buscan identificar emociones transmitidas en el habla, concluyendo con éxito su trabajo, en el que valoran positivamente la capacidad de detectar emociones a través del habla.

Los resultados que ofrece el cuestionario, en relación al reconocimiento sobre la importancia del trabajo emocional como elemento transversal desde cualquiera de las áreas, muestran valores positivos. En concreto, el $94,9 \%$ de la muestra se posiciona afirmativamente a la cuestión sobre si como futuro/a docente, creen que es importante trabajar las emociones, como elemento transversal, desde cualquiera de las áreas. Coincidiendo así con estos resultados, Martínez-Hita (2017) consecuente con el conocimiento que tiene sobre los beneficios que aporta el hecho de fomentar la competencia emocional, defiende en su trabajo una educación emocional entendida como un proceso que permanezca en el tiempo de manera constante, 
entendiendo así que "La formación de estas capacidades emocionales debe formar parte del currículo, [...] Debe existir una sinergia de los contenidos académicos y los emocionales para conseguir el desarrollo de una educación emocional que permita el desarrollo integral de la persona” (p.22). De manera análoga, Ulutaş, İLkay y Ömeroğlu, (2007) demuestran en su trabajo como contribuyen de manera significativa el desarrollo de programas educativos en IE en los niños, al igual que como Torrijos et al. (2018) donde se concluye como los docentes que llevan a cabo su estudio, muestran un incremento en el nivel de motivación durante el desarrollo de la formación en competencia emocional, encontrando útiles los aportes recibidos. Por último, encontramos a Escolar et al. (2017) que afirman haber constatado como paulatinamente ha ido aumentando la oferta formativa para los docentes en los últimos años, debido al interés surgido, debido al conocimiento de estos sobre sus carencias y conscientes de la responsabilidad que tienen a la hora de transmitir, no sólo conocimientos, sino habilidades sociales y emocionales a sus estudiantes.

También se puede ver en la Figura 5, como los resultados son muy positivos en lo relativo a la IE y el uso de la lengua como medio de expresión. Concretamente, el $61,5 \%$ de los estudiantes indican que siempre trabajarán la IE a través del uso de la lengua en el aula. El 56\% de los participantes muestran que tienen disposición a realizar actividades semejantes a la llevada a cabo en el aula. Por último, el $74 \%$ de los futuros docentes de la muestra, considera de vital importancia trabajar la IE en el aula.

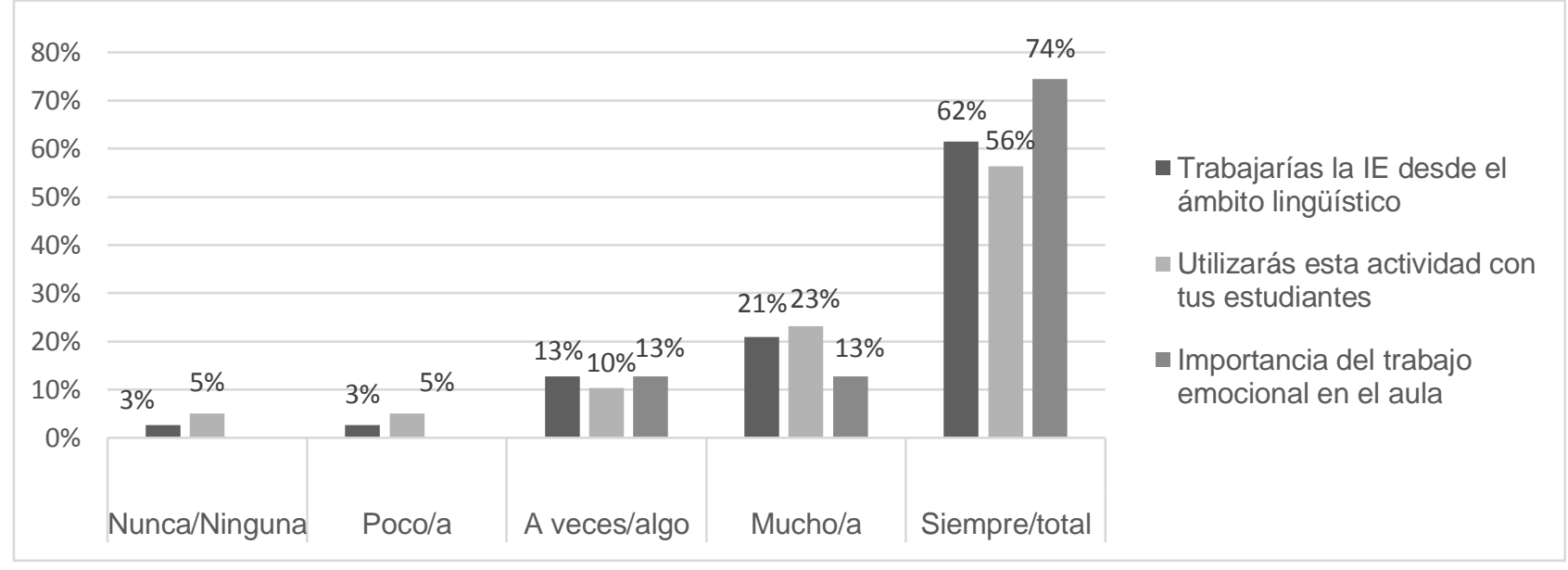

Fig. 5: IE y el uso de la lengua como medio de expresión

De acuerdo con Mestre, Pérez, González, Núñez y Guil (2017) las asignaturas en las que se puede fomentar y potenciar de una manera más clara la comprensión de las emociones son las Ciencias Sociales y Humanidades, ya que estas ofrecen la posibilidad de relacionar los conocimientos a las emociones existenciales para poder entender de mejor manera las emociones. Además, para la regulación emocional refieren una mayor implicación y esfuerzo en el desarrollo cognitivo, así como el lingüístico, social, emocional y moral. Por lo que los resultados obtenidos en este estudio, y la apuesta que se hace de fomentar la competencia emocional a través del uso de la lingüística como forma de expresión y regulación queda refrendada también por estos autores.

\section{CONCLUSIONES}

De los resultados mostrados, de su análisis y de su discusión, se pueden obtener las siguientes conclusiones, sobre el desarrollo de la conciencia emocional en el aula: 1) la competencia emocional cuenta con un alto nivel de interés por parte de los futuros docentes, que creen en los beneficios que posee el trabajo emocional para el desarrollo de las personas; 2) es necesario potenciar la formación previa en el profesorado sobre la competencia emocional, en identificación de las emociones; 3) es significativo el valor que tiene el aspecto lingüístico como medio de expresión emocional, ofreciendo la posibilidad de trabajar la verbalización de las emociones; 4) el trabajo emocional en el aula tiene gran importancia para los futuros docentes; 5) es necesario poder incluir como contenido transversal la educación emocional para mejorar la calidad educativa. Algunas de las principales recomendaciones que han surgido como resultado de la intervención realizada instan a replicar la actividad con una muestra mayor y con un método de muestreo probabilístico que garanticen la generabilidad de los resultados. De manera que, para futuras líneas de investigación se pretenderá poder ampliar la muestra objeto de estudio.

\section{REFERENCIAS}

Alaminos, A., La música como lenguaje de las emociones. Un análisis empírico de su capacidad performativa, http://dx.doi.org/10.14198/OBETS2014.9.1.01, OBETS Revista de Ciencias Sociales, 9(1),15-42 (2014) 
Beck, L., Kumschick, I., y otros dos autores, Relationship between language competence and emotional competence in middle childhood. https://doi.org/10.1037/a0026320, Emotion, 12(3), 503-514 (2012)

Bisquerra, R., La educación emocional en la formación del profesorado, Revista Interuniversitaria de Formación del profesorado, ISSN: 0213-8646, 3(19), 95-114 (2005)

Buenrostro-Guerrero, A.E., y Valadez-Sierra, M.D. y otros cuatro autores, Inteligencia emocional y rendimiento académico en adolescentes. Revista de Educación y Desarrollo, ISSN: 1665-3572, 20, 29-37 (2012)

Cohen, J., Social, emotional, ethical, and academic education: creating a climate for learning, participation in democracy, and well-being, https://doi.org/10.17763/haer.76.2.j44854x1524644vn, Harvard Educational Review, 76 (2), $201-237$ (2006)

Corcoran, R., y Tormey, R., Does emotional intelligence predict student teachers performance?, https://doi.org/10.1016/j.tate.2013.04.008, Teaching and Teacher Education, 35, 34-42 (2013)

Escolar Llamazares, M.C., De la Torre Cruz, T., Huelmo García, J., y Palmero Cámara, C. Educación emocional en los profesores de Educación Infantil: aspecto clave en el desempeño docente. http://doi.org/10.18172/con.2994 Contextos Educativos, 20, 113-125 (2017)

Fabio, A., y Palazzeschi, L., Emotional intelligence and self-efficacy in a sample of Italian high school teachers, http://doi.org/10.2224/sbp.2008.36.3.315, Social Behavior and Personality: an international journal, 36(3), 315-326 (2008)

Giraldo, G. Los procesos de lectura y escritura en la Inteligencia, http://dx.doi.org/10.15765/plnt.v7i13.146, POLIANTEA Educación, 7(13), 205-218 (2011)

Hawkey, K., Emotional intelligence and mentoring in pre-service teacher education: a literature review, http://doi.org/10.1080/13611260500493485, Mentoring \& Tutoring: Partnership in Learning, 14(2), 137-147 (2006)

Jennings, P., y Greenberg, M., The Prosocial classroom: teacher social and emotional competence in eelation to student and classroom outcomes, https://doi.org/10.3102/0034654308325693, Review of research in Education, 79(1), 491-525 (2009)

Llamazares, M.C., De la Torre, T., Huelmo, J., y Palmero, C. Educación emocional en los profesores de educación Infantil: aspecto clave en el desempeño docente, https://doi.org/10.18172/con.2994, Contextos Educativos, 20, 113-125 (2017)

Marín-Moya M.C., Ruiz, M.J., Sánchez-Abril M., y Pagán, J., Desarrollando la Inteligencia emocional a través de cuentos populares, Experiencias y recursos de innovación en Educación Infantil by Gómez, C.J., e Izquierdo, T. (Eds.), Universidad de Muria: Servicio de Publicaciones, pp.123-132, Murcia, España, (2015)

Martínez-Hita, M. Educación emocional. El cuento como herramienta para su desarrollo, https://doi.org/10.14422/pym.i370.y2017.003, Padres y Maestros, 370,18-22 (2017)

Mestre, J.M., Pérez N., y otros 3 autores, El desarrollo de la inteligencia emocional a través de la optimización de las capacidades cognitivas a través de la Educación Obligatoria, https://doi.org/10.18172/con.3023, Contextos Educativos, 20, 50-75 (2017)

Mujica, F., Inostroza, C., y Orellana, N., Educar las emociones con un sentido pedagógico: un aporte a la justicia social, https://doi.org/10.15366/riejs2018.7.2.007, Revista Internacional de Educación para la Justicia Social, 7(2), 113-127 (2018)

Ozáez, M.T., Inteligencia emocional en educación primaria. Revista Internacional de Apoyo a la Inclusión, Logopedia, Sociedad y Multiculturalidad, ISSN: 2387-0907,1(3), 51-60 (2015)

Ozlem, A., Is Emotional intelligence or mental intelligence more important in language learning? Journal of Applied Sciences, ISSN 1812-5654, 6(1), 66-70 (2006)

Páez, M., y Castaño, J., Inteligencia emocional y rendimiento académico en estudiantes universitarios. Psicología desde el Caribe, ISSN: 0123-417X, 32(2),.268-285 (2015)

Palomera, R., Fernández-Berrocal, P., y Brackett, M., Emotional intelligence as a basic competency in pre-service teacher training: some evidences, Electronic Journal of Research in Educational Psychology, ISSN: 1696-2095, 15(6), 437-454 (2008)

Pérez-Escoda, N., Filella, G., Alegre, A., y Bisquerra, R. (2012). Desarrollo de la competencia emocinoal de maestros y alumnos en contextos escolares, Electronic Journal of Research in Educational Psychology., ISSN: 1696-2095, 10 (3), 1183 -1208 (2012)

Petrides, k., Sangareau, Y., y otros dos autores, Trait emotional intelligence and children's peer relations at school, https://doi.org/10.1111/j.1467-9507.2006.00355.x, Social Development, 15(3), 537-547 (2006)

Resnicow, J., Salovey, P., Repp, B., Is recognition of emotion in music performance an aspect of emotional intelligence?, Music Perception: An Interdisciplinary Journal, 22(1), 145-158 (2004)

Swanepoel, S., y Britz, L., Emotional intelligence and academic performance, https://doi.org/10.29086/25195476/2017/sp20a9, Alternation Journal, 20, 171-188 (2017)

Soriano, C., El lenguaje de las emociones. Panorama actual de la ciencia del lenguaje, In M. C. Horno Chéliz, I. Ibarretxe Antuñano \& J.L. Mendívil Giró (Eds.), pp. 243-259, Zaragoza: Prensas de la Universidad de Zaragoza, España, (2016) 
Sucaromana, U., Contribution to language teaching and learning: a review of emotional intelligence, http://dx.doi.org/10.5539/elt.v5n9p54, English Language Teaching, 5(9), 54-58 (2012)

Schellenberg, E., Music lessons, emotional intelligence and IQ., Music Perception Interdisciplinary Journal, 29(2), 185-194 (2011)

Torrijos, P., Martín Izard, J.F., y Rodríguez, M.J., La educación emocional en la formación permanente del profesorado no universitario, Revista de Currículum y formación del profesorado, ISSN 1989-6395, 22 (1), $579-597$ (2018)

Trimmer, C.G., y Cuddy, L.I, Emotional intelligence, not music training, predicts recognition of emotional speech prosody, https://doi.org/10.1037/a0014080, Emotion, 8(6), 838-849 (2008)

Ulutaş, İ., y Ömeroğlu, E., The effects of an emotional intelligence education program on the emotional intelligence of children, https://doi.org/10.2224/sbp.2007.35.10.1365, Social Behavior and Personality: an international journal, 35(10), 1365-1372 (2007) 